\title{
Geometría y traza de escaleras góticas. Las escuadras como ábacos en la construcción de los caracoles de la catedral de Tortosa
}

\section{Geometry and traces of Gothic stairs. The squares as instrumental abacuses in construction spirals stairs of Tortosa Cathedral}

J. Lluis i Ginovart ${ }^{(*)}$

RESUMEN

Las escaleras de caracol en el período gótico han sido uno de los mayores exponentes del conocimiento de los magister operis. En él se concentran los saberes aritméticos y geométricos del constructor medieval. Para su construcción se utilizan los gabarits, que necesitan el concurso de la división poligonal de la circunferencia. Los polígonos regulares de 11, 13, 14, 17 y 19 caras no aparecen en los Elementa de Euclides, ni en el Almagesto de Tolomeo, ni en las escasas Practica Geometriae de la época. Estos polígonos pueden ser construidos mediante la utilización de una escuadra de dos brazos, con el apoyo reiterado de los catetos sobre la hipotenusa, creando así una rotación del instrumento. Las escuadras construidas por métodos aritméticos o geométricos pueden ser utilizadas como un ábaco, el cual permite resolver con suficiente exactitud la división de la circunferencia. Las escaleras de San Pedro (1383) y Santa Catalina (1424) de la catedral de Tortosa han sido trazadas mediante estos instrumentos.

Palabras clave: Catedral Tortosa; geometría medieval; tratados históricos; polígonos regulares; escuadras.

\section{ABSTRACT}

In the Gothic era, spiral staircases were one of the greatest showpieces of the magister operis knowledge. They were the focus of arithmetic and geometric medieval constructor knowledge. Their construction involved the use of templates known as gabarits, which required the polygonal division of the circumference. These regular 11-, 13-, 14-, 17- and 19-sided polygons do not appear in the Euclid's Elementa or the Tolomeo's Almagest, or in the few Practica Geometriae of the time. These polygons can be constructed by using a square with two arms, supporting repeatedly the legs on the hypotenuse, a rotation of the instrument. Squares constructed using arithmetic or geometric methods must always be calibrated and can therefore be used as an abacus, which enables the circumference to be divided with sufficient accuracy. San Pedro (1383) and Santa Catalina staircases (1424), of Tortosa's cathedral, have been drawn by the instruments explained previously.

Keywords: Tortosa Cathedral; medieval geometry; historical treatise; regular polygon; square.

(*) Universitat Rovira i Virgili. Reus (España).

Persona de contacto/Corresponding author: josep.lluisg@urv.cat (J. Lluis i Ginovart)

Cómo citar este artículo/Citation: Lluis i Ginovart, J. (2016). Geometría y traza de escaleras góticas. Las escuadras como ábacos en la construcción de los caracoles de la catedral de Tortosa. Informes de la Construcción, 68(541): e132, doi: http://dx.doi.org/10.3989/ ic.15.035.

Licencia / License: Salvo indicación contraria, todos los contenidos de la edición electrónica de Informes de la Construcción se distribuyen bajo una licencia de uso y distribución Creative Commons Reconocimiento no Comercial 3.o. España (cc-by-nc). 


\section{INTRODUCCIÓN}

La nueva catedral gótica de Tortosa sustituyó a la seo románica, la ecclesiam vetulam, que había sido consagrada en 1178 , tras la reconquista de la ciudad en 1148. La tipología de la arquitectura gótica del siglo xIv, se adaptará a la nueva liturgia del Prochiron vulgo rationale divinorum officiorum (1291) de Guillermo de Durando (1230-1296), con un ábside con capillas radiales (Figura 1).

Tras el inicio de la obra gótica (1345), se produjo una paralización de la fábrica debida tanto a la peste (1347), como a la Guerra de los dos Pedros (1356-1369). La obra se reanudó con la construcción del cinturón de las nueve capillas radiales, situadas alrededor de la catedral románica. Las nueve capillas radiales, de planta cuadrada y con bóvedas de crucería, fueron construidas de forma correlativa, desde el evangelio a la epístola (1383-1424). La sección teórica de las claves de las bóvedas fue rematada a una altura de 45 palmos (1) (Figura 2).

El ábside empieza con la construcción de la capilla de San Pedro (1383) y finalizará con la de Santa Catalina (1424). En los extremos de estas capillas radiales se construyen sendas escaleras de caracol del tipo vis a pourtant noyau, caracol de husillo en español, o caragol de botó redó en lengua catalana. Ambas sirvieron de acceso y apoyo para la construcción del deambulatorio, el cual fue construido de forma simétrica sobre el presbiterio, siendo de gran utilidad para el apeo y servicio auxiliar de la construcción de la nueva fábrica gótica (2).

En el Dictionaire raissoné de l'architecture, Eugene Violletle-Duc (1814-1879) situaba el origen de l'escalieres à vis en la tradición constructiva romana. Las primeras escaleras de caracol francesas se construyeron sobre bóveda de piedra, como la del monasterio de Eu en Normandia (c. 1150) (3). Por su parte, August Choisy (1841-1909) explicaba en L'art de bâtir chez les bizantins otras tipologías de caracoles construidos sobre hojas de ladrillo (4).

En la construcción de escaleras hace falta un conocimiento aritmético para determinar el número total de escalones $\left(n_{i}\right)$ y salvar la altura de paso de una persona $\left(n_{0}\right)$. Aunque también es necesario el conocimiento geométrico para dividir la circunferencia en $\left(n_{o}-1\right)$ partes, en función del número de

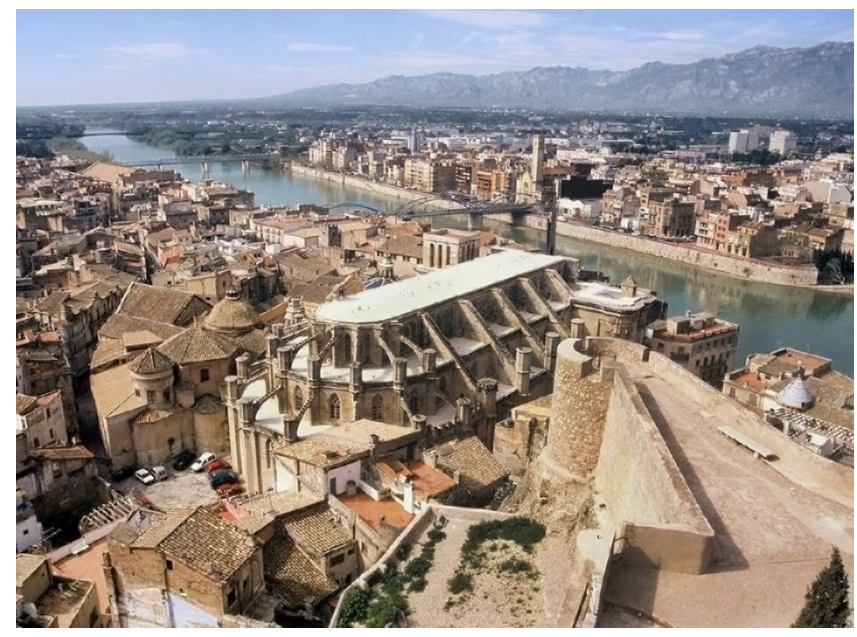

Figura 1. Catedral Santa María de Tortosa. peldaños de la rosca $\left(n_{o}\right)$. Por tanto, la construcción de escaleras de caracol, es uno de los mayores exponentes del conocimiento matemático de los magister operis.

El objetivo de la investigación es determinar la traza de las escaleras de caracol mediante el apoyo de escuadras. Éstas son utilizadas como plantillas de los escalones. Para el desarrollo de esta tesis se toma como referencia la metodología utilizada en el álbum de Villard de Honnecourt (ca. 1220-1250) (BnF. ms. fr. 190093, fol. 20 v), en la cual, con un movimiento de una plantilla, se construye la base de una torre pentagonal. Ésta hipótesis ha sido estudiada por R. Bechmann (5). Otra referencia será la tumba de Saint-Ouen (ca.130o), donde existe una ábaco que ha sido asimilado a la construcción de polígonos regulares inscritos en una circunferencia, tema tratado por M. T. Sarrade (6). Otra alusión directa a estas metodologías es la de Diego López Arenas (+c. 1640) en la Primera y segunda parte de la carpintería hecho por Diego López de Arenas (1619), donde se define el cartabón como instrumento para la traza de polígonos regulares, hipótesis constatada por E. Nuere (7). Otra tesis para la construcción de estas escaleras sería la división angular, utilizada para la construcción de astrolabios en la tradición de algunas Practica Geometiae (1125-1130) como la de Hugo de San Víctor (ca. 11001141) (8), o la de Dominicus de Clavasio de (1346) (9).

\section{LAS ESCALERAS GÓTICAS DE CARACOL}

Existen diferentes tipologías de escaleras en función del número de peldaños $\left(n_{0}\right)$, ya sea en las trazas de la época gótica, o en los tratados tardogóticos. Son casi 200 los ejemplos de trazas de escaleras semejantes a las escaleras de la catedral de Tortosa (Figura 3). Es el caso de los proyectos de la escalera
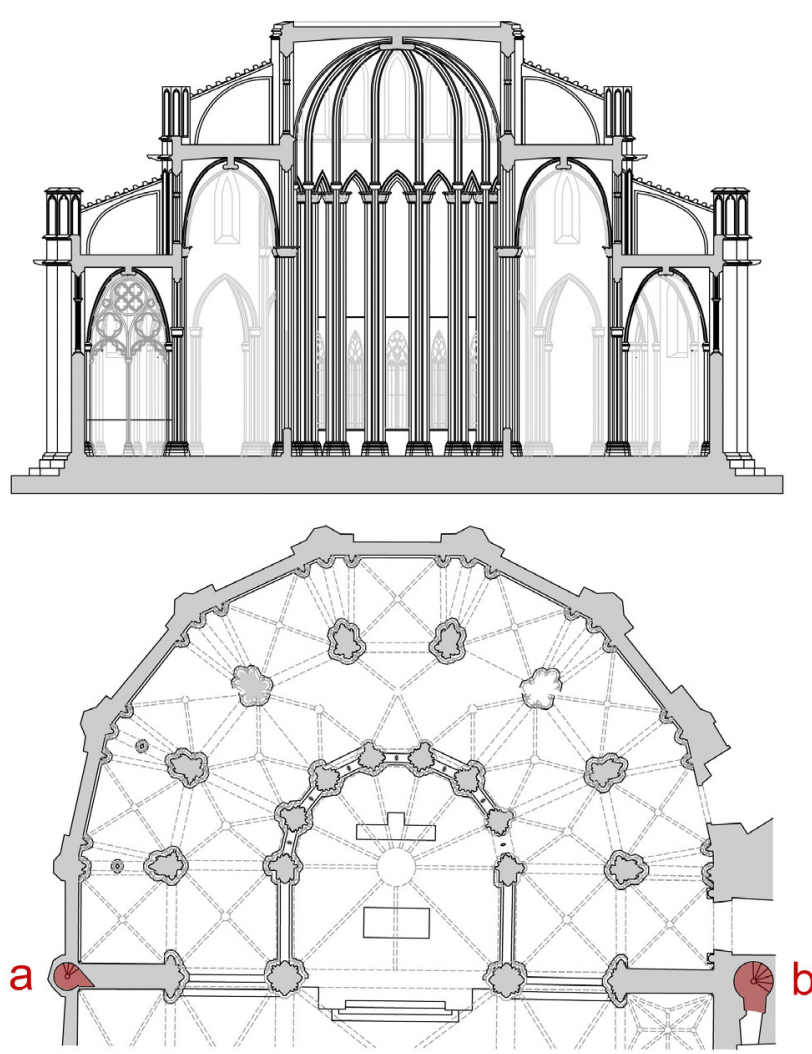

Figura 2. Ábside catedral Santa María de Tortosa (1383-1441). a) Escaleras de San Pedro (1383). b) Santa Catalina (1424). 
de la catedral de Estrasburgo (c.1400) $\left(\mathrm{ABK} \mathrm{n}^{0}\right.$ 16953) con 16 rellanos (10), o la de Viena (c.1500) (ABK no 16.855) que se divide en 15 partes (11). En el catálogo del Maestro WG (1560-1572) de Frankfurt, la traza (WG 26-1) está divida en 14, la (WG 26-2) en 12 y la (WG 35) en dieciséis mesetas (12).

En la tratadística aparece la escalera de espiral con 12 peldaños del Underweysung der Messung (1525) de Albrecht Dürer (1471-1528) (13). La traza de vis de Saint Gilles con 24 rellanos de Philibert de l'Orme (1510-1570) publicada en la L'architecture (1576) (14). En el Libro de trazas de cortes de Piedras (c.1580), atribuido a Alonso de Vandelvira (15441626), el caracol de emperadores tiene 28 rellanos (15). En el manuscrito Cerramientos y Trazas de Montea (c. 1600) de Ginés Martínez de Aranda (1556-1620), se traza el caracol de usillo con 12 divisiones (fol.222), y en el doble, se construye con 18 peldaños (16). En la Vertaderas traçes del Art de picapedrer (1653) de Josep Gelabert (1621-1668) los ejemplos del caracol son: el de botó redó (fol. 42v-43r), con un polígono de 11 lados, el de botó fa pilar entorxat, con 16 lados (fol. 43v-44r), el de ull ubert (fol. 44v - 45v) con 12 escalones, botó redó $y$ ullobert (fol. 45v-46r) con 17 peldaños. Finalmente el dos cargols qui ballen per dins un redó (fol. 46v-47r), construidos con 10, 11 o 12 rellanos (17). a)

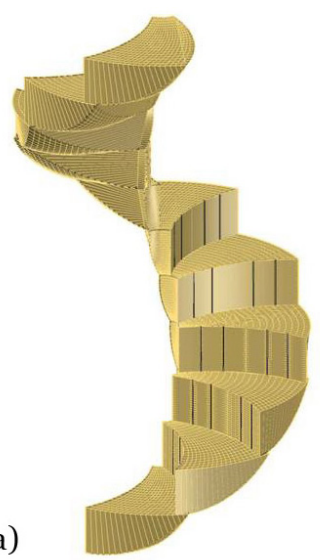

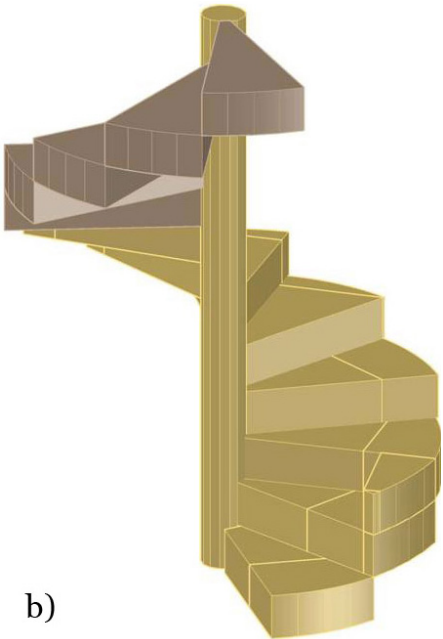

b)
Estas escaleras de caracol están inscritas mediante polígonos regulares de 10, 12, 15, 16, 18, 24, 28 lados. Éstas tienen como base de su construcción geométrica, las figuras del cuadrado, pentágono, hexágono y octógono, los cuales se pueden identificar directamente en los Elementa de Euclides (326$265 \mathrm{aC})$, traducidos por Adelardo de Bath (1075-1166), y en el Almagesto de Ptolomeo (c.85-165), trasladado por Gerardo de Cremona (1114-1187) ${ }^{1}$. Sin embargo, alguno de los polígonos en que se divide el caracol de 11, 13, 14, 17 o 19 lados, no aparecen en estas referencias cultas.

En el caso de la catedral Tortosa, la escalera de caracol de San Pedro (1383) está dividida en 10 partes, y su construcción geométrica tiene como base el pentágono; mientras que para la construcción de la escalera de Santa Catalina (1424), con 13 lados, no se tiene referencia directa en los textos medievales. Por tanto, el maestro medieval debía tener algún método para encajar los peldaños en el interior de su escalera de caracol. A esta dificultad geométrica y aritmética hay que añadir la dificultad de la talla y estereotomía, como es el caso de la construcción de la vis de Saint Gilles (18), la escalera de husillos con machón helicoidal (19), o las de caracol con ojo (20). Por ello, para el trazado de estas escaleras de caracol, es necesaria la utilización de una plantilla, construida mediante una escuadra.

\section{LA BELLEZA CLÁSICA DE LAS ESCUADRAS}

Las escuadras están definidas en el Timaeus de Platón (c.429-437 aC) (21), donde se determina el triángulo como elemento básico de la geometría (Tim. 53,c-d). Al triángulo rectángulo isósceles, le asignará el mayor grado de belleza $\left(45^{\circ}-90^{\circ}-45^{\circ}\right)$. A partir del escaleno $\left(30^{\circ}-90^{\circ}-60^{\circ}\right)$ se genera el triángulo equilátero (Tim. 54,d-e), y el cuadrado a partir del isósceles (Tim. 55,b) (Figura 4).

Aristóteles (384-322 aC) definirá, en el Metaphysicorum, el triángulo como la unidad elemental, el Uno, (Met. L.X, $2,1054^{\mathrm{a}}$ a, 1-5), conocerá la propiedad de los triángulos, en la que la suma de sus ángulos es igual a $180^{\circ}$. Por ello construirá ángulos rectos a partir del arco capaz (Met. L.IX, 10, 1051 a , 25-30) (22). Será Euclides (326-265 aC) en sus Elementa (L.I. 47)] quién demostrará el teorema del triángulo rectángulo, $a^{2}=b^{2}+c^{2}$, donde $a=1 / 2\left(b^{2}+1\right)$ y $c=1 / 2\left(b^{2}-1\right)(23)$.

Figura 3. Escaleras de caracol. a) San Pedro (1383). b) Santa Catalina (1424).

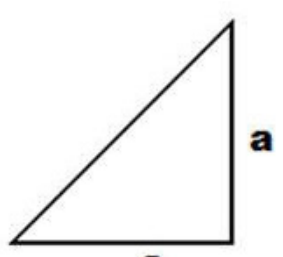

a

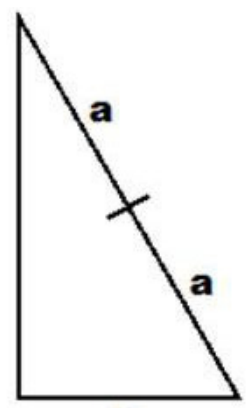

a

Tim. 53 c-d

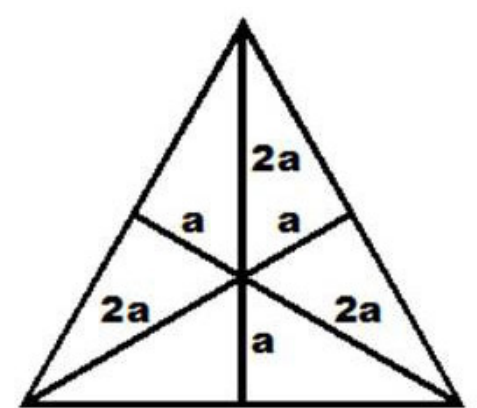

Tim. 54 d-e

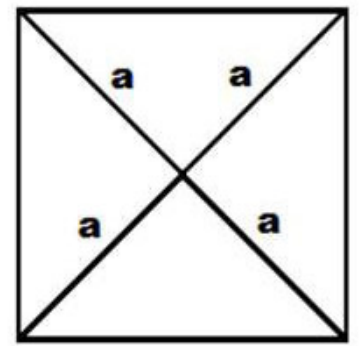

Tim. 55 b

Figura 4. Escuadra de Platón.

\footnotetext{
${ }^{1}$ En el libro IV de los Elementa de Euclides aparecen: el triángulo (IV.5), cuadrado (IV.6- 9), pentágono (IV.11-14), hexágono (IV.15), pentadecágono (IV.16). También lo hacen: el cuadrado, pentágono, hexágono, decágono y dodecágono, en el Almagesto (LI, IX) de Ptolomeo.
} 
Vitrubio (c. 8o- c.20 aC) en su De Architectura (c. 15), recurrirá a Platón, definiendo el cuadrado de lado 10 con sus diagonales (L.IX.2) y a Pitágoras $(3,4,5)$ (L.IX.2) (24). Por su parte, Cetio Faventino (f. 350) en el Artis architectonicae privatis usibus adbreviatus liber, dice que la escuadra es el descubrimiento del principio de la proporcionalidad, utilizando la terna $(2,2,2+10 / 12)(25)$. Escuadra recogida también en las Etymologiarum de San Isidoro de Sevilla (560636) (Etm.XIX.18.1) (26), y transmitida al Libro XXI.XI del De rerum naturis, de Rabanus Maurus (c.780-856) (27).

\section{LAS ESCUADRAS GÓTICAS}

Formalmente, las escuadras clásicas tienen brazos perpendiculares entre sí, y a la vez paralelos, y por tanto, con dos ángulos, interno y externo de $90^{\circ}$. A partir del siglo xiII, aunque se siguen utilizando la escuadras clásicas, aparecen las conocidas como d'ongletes (28). Estas escuadras tienen los brazos divergentes, donde el ángulo externo es recto, mientras que el interno no lo es. Estas escuadras pueden ser utilizadas tanto a modo de ábacos, como también para la estereotomía de la piedra (29). Existen así diferentes formas: la conocida como canónica, con ángulos de $30^{\circ}-60^{\circ}$ de Hugues Libergier (c.1263) (30) (Figura 5a), la pitagórica $(3,4,5)$ de Niederhaslach (31) (Figura 5b), las de doble cuadrado, como la Life of the Offas (c.1236) (32), o la de la catedral de Gerona (33) (Figura $5 \mathrm{c}$ ). Otras con coordenadas decagonales $\left(36^{\circ}-54^{\circ}\right)$, como la de la catedral de Poitiers (34) (Figura 5d), o escuadras que tienen como base el triángulo áureo $\left(31,72^{\circ}-58,20^{\circ}\right)$ (35) (Figura 5e).

En el cuaderno de Villard de Honnecourt (c.1175-1240), (BnF. ms. fr. 190093, fol. 20 r), aparecen tres representaciones de escuadras de brazos no convergentes, utilizadas para la talla de salmeres de una bóveda (f), la construcción de dovelas (i), y otra para la duplicación de volúmenes (q) (36). La escuadra se utiliza también como elemento auxiliar para medir alturas que son inaccesibles (fol. $20 \mathrm{v}$ ) (37).

En otro contexto, Berand Boysset (1355-1415) en La siensa de atermenar (1401), dedicará una parte a la construcción de la escuadra. Un método lo realiza con regla y compás (CBM. 237, fol. $215 \mathrm{r}, 216 \mathrm{v}$ ) (Figura 6a), mientras que el otro lo hace sin instrumentos (CBM 327, fol. $216 \mathrm{v}$, fol $220 \mathrm{v}$ ) (Figura 6b) (38).

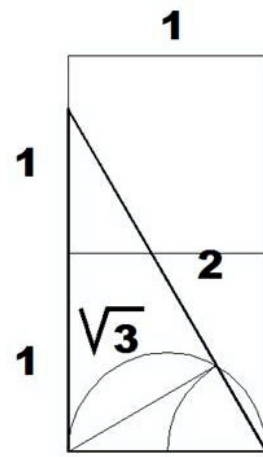

$\left(30^{\circ}, 60^{\circ}\right)$

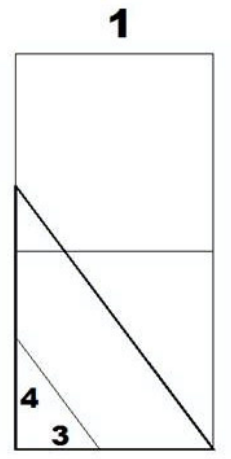

$\left(36^{\circ} 87,53,13^{\circ}\right)$

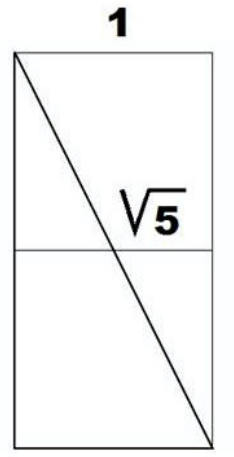

$\left(26.57^{\circ}, 63.43^{\circ}\right)$

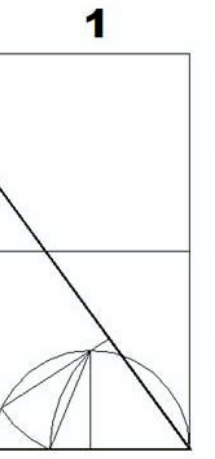

$\left(36^{\circ}, 5^{\circ}\right)$
1

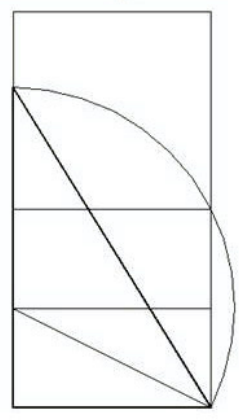

$\left(31,72^{\circ}, 58,28^{\circ}\right)$

a

b

c

d

Figura 5. Tipologías escuadras góticas.
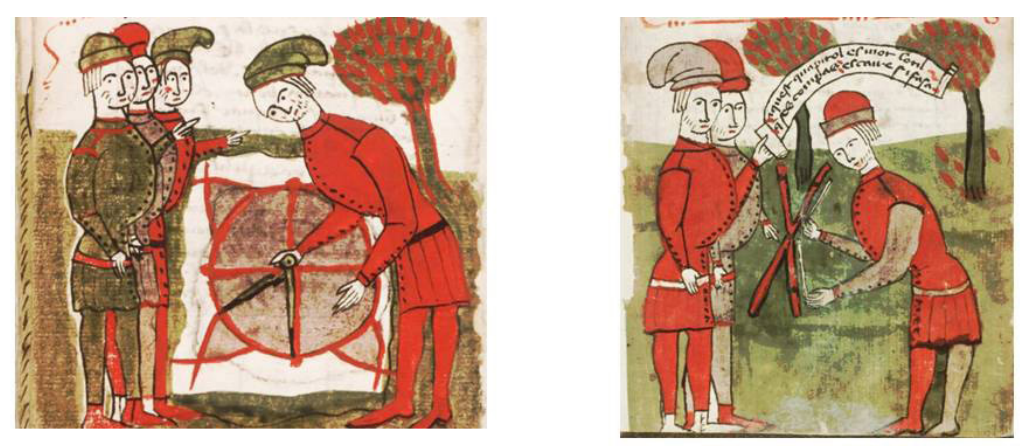
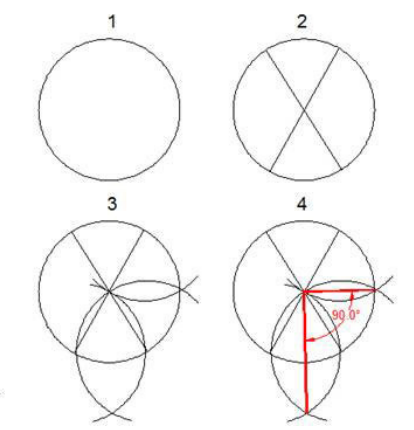

2

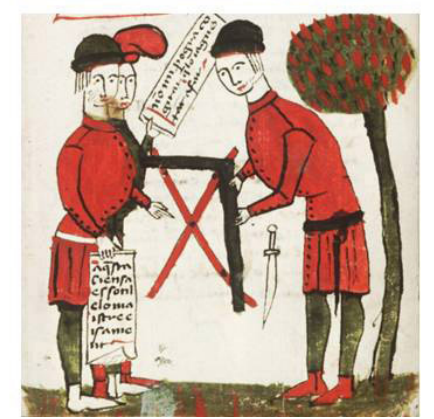

Figura 6. Construcción de una escuadra Berand Boysset (1401). 
La primera geometría escrita y difundida fuera de las logias, la Geometrie Deutsch (1488) de Matthäus Roriczer (+c. 1495), construye la escuadra con una operación combinada de compás con dos diámetros y tomando tres puntos extremos, en la tradición de Boyssey (39). Albrecht Dürer (1471-1528) en el Underweysung der Messung (1525) construye la escuadra a modo de Roriczer (40).

\section{TRAZADOS DE POLÍGONOS CON ESCUADRAS POR ROTACIÓN}

La construcción de la base de la torre del Villard de Honnecourt (fol.20.r.c) (Figura 7a), Par chu portait om one toor a chinc arestes ha sido interpretada por M. T. Sarrade y R. Bechmann, como la construcción de un pentágono mediante una escuadra que va situándose por los puntos medios y sextos, (1/3-0,77), creando así los vértices del polígono regular inscrito en la circunferencia. Hemos reconstruido el proceso de estos autores mediante una escuadra de proporción $1 / 3$, con (100 u) en 4 lados y el de cierre $(102,61 \mathrm{u})$, y con un ángulo de giro $\left(108,43^{\circ}\right)$ (Figura $\left.7 \mathrm{~b}\right)$.
Otro método que proponemos se realiza con una escuadra de proporciones de (2/6), con la que se situará una alineación entre el segundo punto de un cateto y el extremo del otro, colocándose sobre esta alineación el nuevo lado del pentágono (Figura 7c). Una solución similar es situar la escuadra en los vértices, apoyando reiteradamente la escuadra sobre la hipotenusa (Figura $7 \mathrm{~d}$ ). Ambas soluciones son coincidentes con los resultados obtenidos por Sarrade y Bechmann.

Otra tesis es la que se puede extraer del ábaco de la tumba de Saint-Ouen, dónde Sarrade plantea la posibilidad de construir polígonos regulares. La hipótesis comprueba que en el punto (a) se determina la diagonal de un cuadrado, en (b) la diagonal de un rectángulo cuya relación es de (4/5), en (c) el lado de un triángulo equilátero y sobre (d) la pendiente de una recta de un tercio (Figura 8). Estas proporciones (a), (b), (c), (d), pueden ser expresadas como (4/4), (4/5), (4/7) y (4/12), pero también en base doce, como el cuadrante de las sombras de un astrolabio; (4), (6+6/7), (9+1/6), (12) (Figura 8).

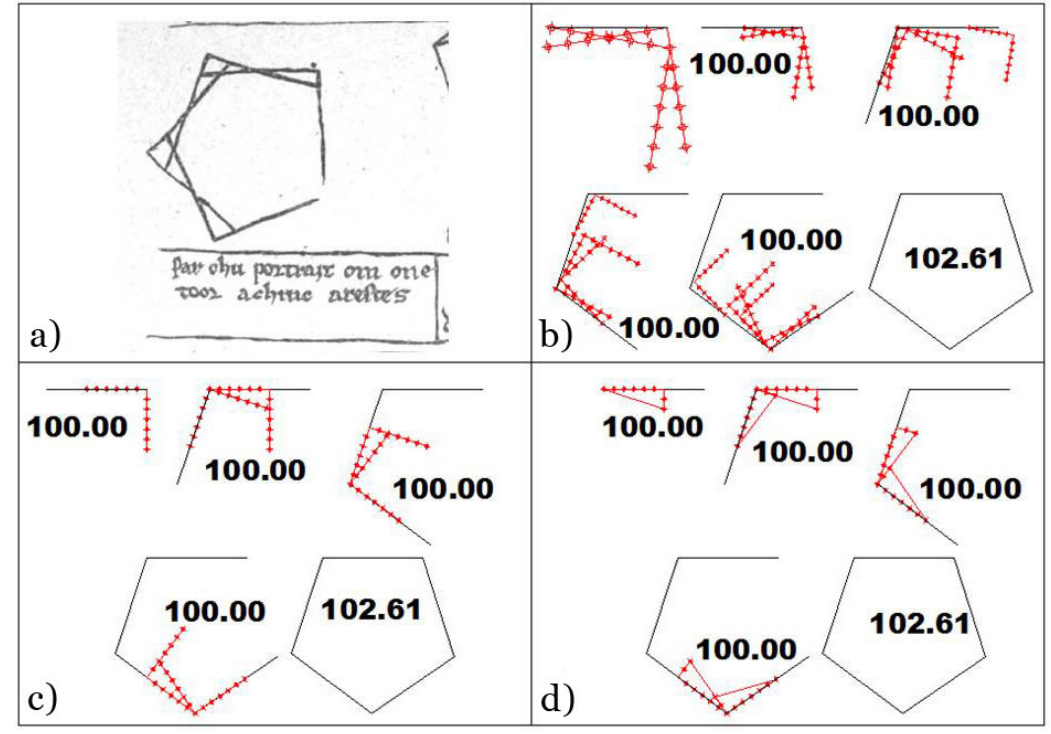

Figura 7. Interpretacción del Villard de Honnecourt (fol. 20 r (c).
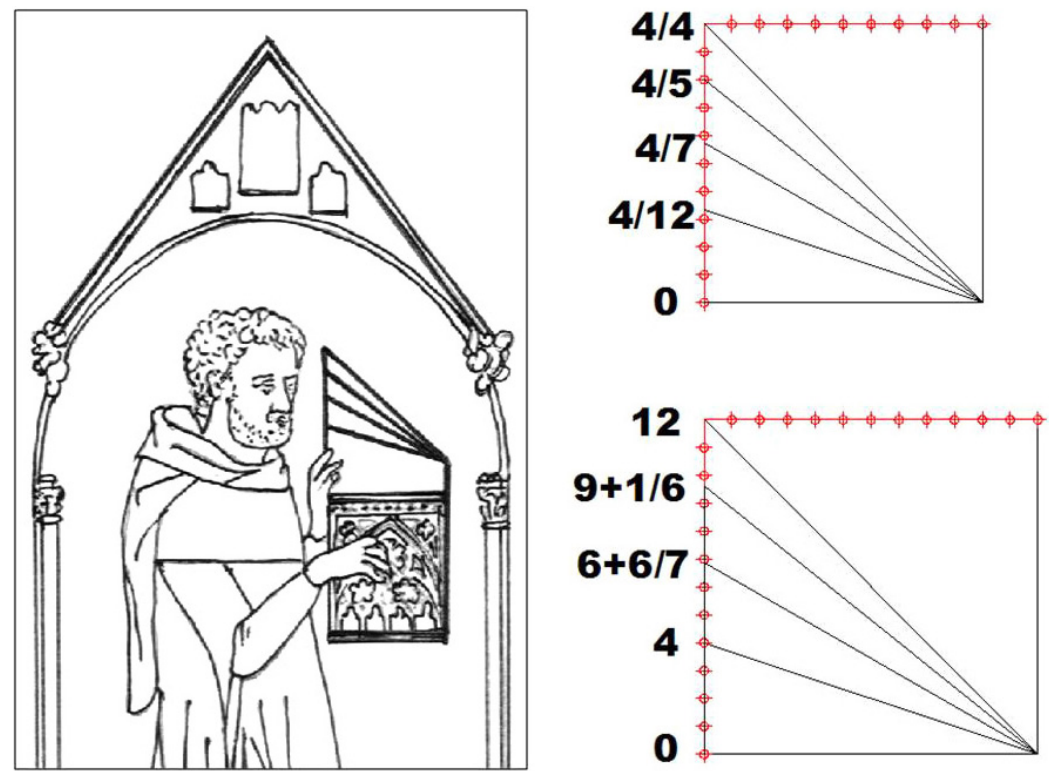

Figura 8. Reinterpretación tumba de Saint-Ouen. 
Con una escuadra (100 u) podemos operar, de modo que su posición relativa vaya girando en el extremo del cateto, haciendo así que la escuadra quede alineada con la hipotenusa. Si tomamos la escuadra isósceles y orientamos una nueva sobre su diagonal, obtendremos un cuadrado. Si repetimos el mismo proceso con las escuadras (100-100/3) obtenemos un pentágono, con la aproximación a la escuadra escalena de (100-40/7) obtenemos un hexágono, con la (100-80) obtenemos el heptágono, con la isósceles (100-100), el octógono. En el caso del cuadrado los cuatro lados tienen la misma medida (100), al igual que los ocho del octógono (100), por lo tanto la construcción es exacta. La construcción de las otras figuras es aproximada: en el pentágono, 4 lados (100) y el cierre $(102,61)$, en el hexágono, 5 lados $(100)$ y el otro $(97,68)$ y en el heptágono 6 lados de (100) y el de cierre $(101,12)$ (Figura 9).

Así, este método sugiere la aproximación de los principales polígonos regulares, de acuerdo con la Tabla 1, donde (l) es el lado desigual, $\left(\alpha^{\circ}\right)$ el ángulo desigual y $(\varepsilon \%)$ el error relativo frente al polígono regular.

\section{LA ESCALERA DE SAN PEDRO (1383) Y LA FIGURA DEL PENTÁGONO}

Tras el comienzo de la obra gótica iniciada en 1346, la construcción se reanudó por la parte de la torre septentrional de la Capilla de San Pedro en 1375, empezando así la construcción

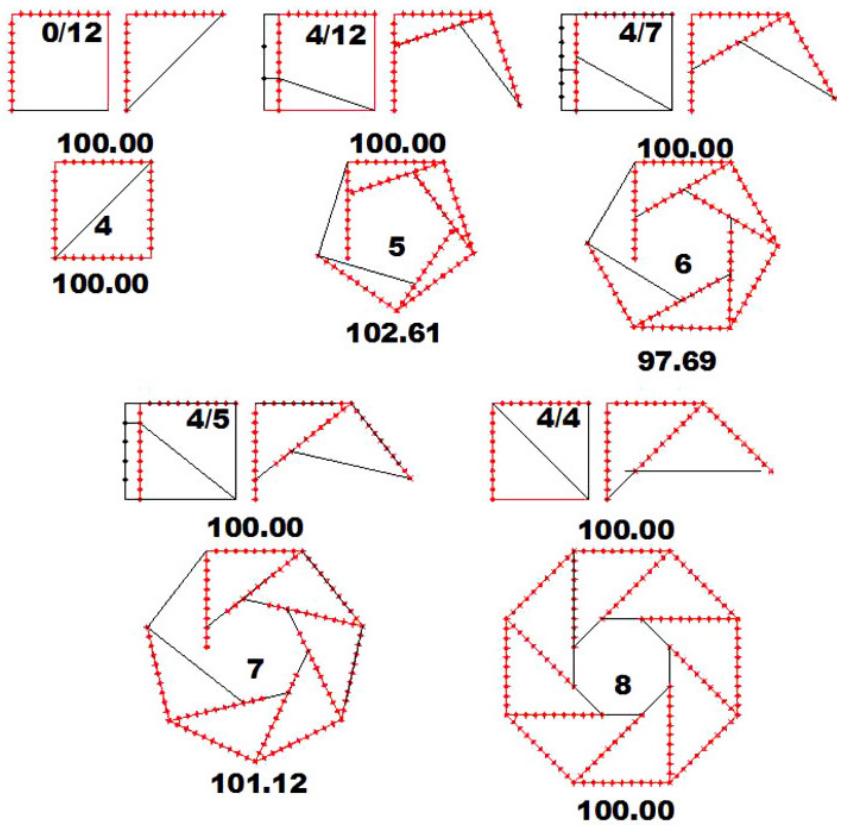

Figura 9. Construcción de polígonos regulares mediante rotación de escuadras. del ábside radial. La escalera de caracol tuvo que ser terminada en el momento que fue cubierta la capilla de San Pedro en el año 1383, por Joan Mayni (41). Años más tarde El rey Martín I (1356-1410) solicita al maestro de la catedral de Tortosa, una mostra de la escalera de caracol de la torre de San Pedro (42) (Figura 10). El desarrollo de la escalera helicoidal de San Pedro es caragol d'ull obert, de planta circular de 144,00 cm, con escalones en forma de lúnula y la sección de su alzado resuelta a modo de elipsoide. Este modelo comporta una mayor comodidad a la hora de permitir la circulación vertical, mejorando considerablemente su accesibilidad. La escalera tiene un paso de rosca de 11 tabicas de 25,00 cm y diez rellanos de medidas de su trapecio de $(5,30 ; 63,00$; 44,50 y 63,00 cm), con un ojo de 17,00 cm de diámetro.
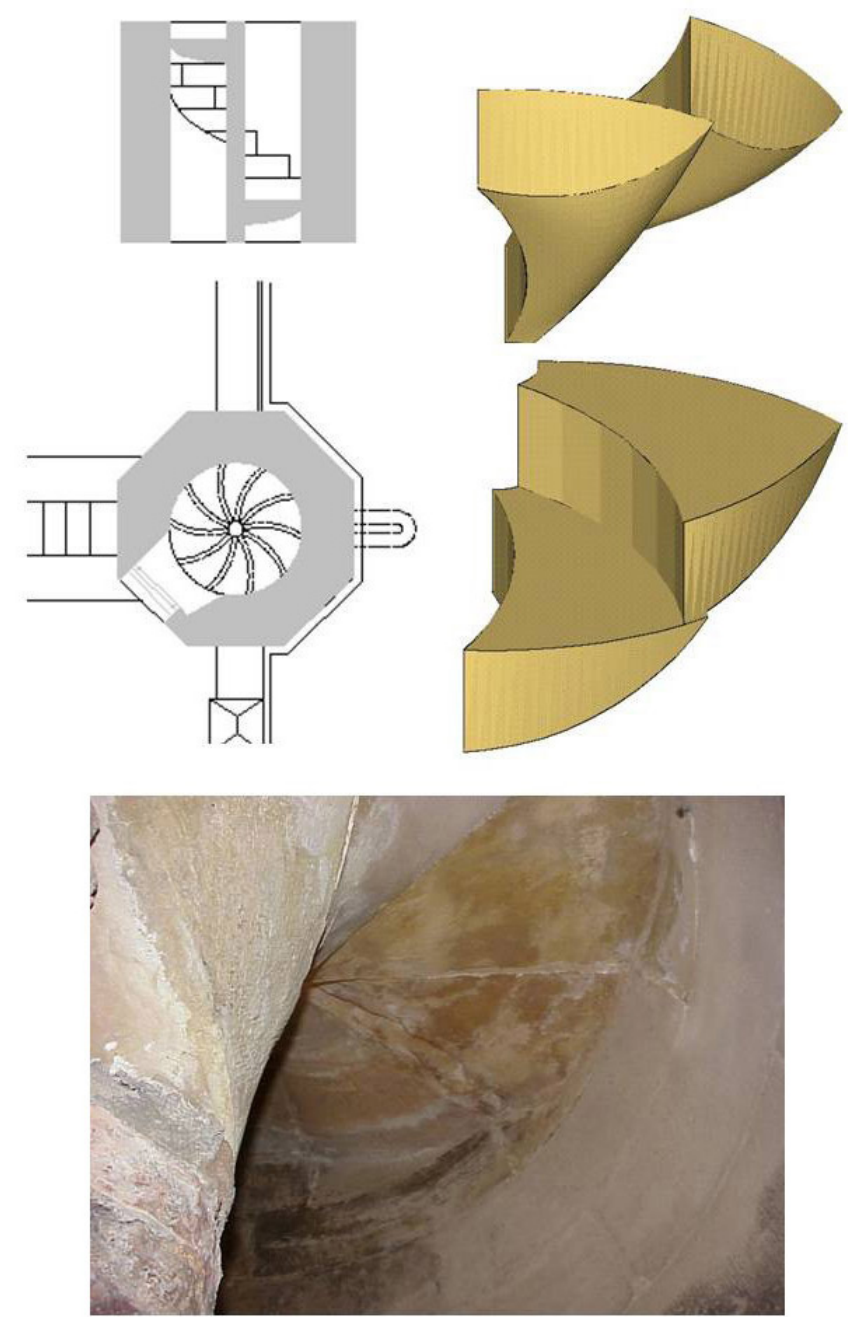

Figura 10. Caragol de San Pedro catedral Tortosa (1383), diámetro $144 \mathrm{~cm}$.

Tabla 1. Interpretación ábaco tumba de Saint-Ouen.

\begin{tabular}{|c|c|c|c|c|c|c|c|}
\hline & & 1 & $l_{i}$ & $\varepsilon \%$ & $\alpha^{\mathbf{o}}$ & $\alpha_{i}^{o}$ & Polígono regular \\
\hline $4 \div 0$ & $100 \div 0$ & 100,00 & 100,00 & 0,00 & 90,00 & 90,00 & Cuadrado \\
\hline $4 \div 12$ & $100 \div 400 / 12$ & 102,61 & 100,00 & 2,61 & 108,43 & 108,00 & Pentágono \\
\hline $4 \div 7$ & $100 \div 400 / 7$ & 97,69 & 100,00 & 2,31 & 119,74 & 120,00 & Hexágono \\
\hline $4 \div 5$ & $100 \div 80$ & 101,12 & 100,00 & 1,12 & 128,66 & 128,57 & Heptágono \\
\hline $4 \div 4$ & $100 \div 100$ & 100,00 & 100,00 & 0,00 & 135,00 & 135,00 & Octógono \\
\hline
\end{tabular}


La construcción geométrica de la escalera tiene como base el pentágono. Esta figura apareció en la primera edición española de los Elementa de Euclides (IV.11-14), de Rodrigo Zamorano (1542-1623) (43) (44), y en la del Almagesto (LI. X) de Jorge de Trapezuntio (1395-1486) (45) (46). También está presente en la tradición árabe de Abū al-Wafā Al-Būzjānī (940-998) en el Kitāb fā mā yaḥtāju al-șāni min al-a'māl al-handasiyya (Libro de las construcciones geométricas que son necesarias para artesanos) (c.9931008) (47) (48).

En la cultura tardogótica, la figura del pentágono aparece resuelta en la Geometrie Deutsch (1488) (P2.fol.2r) (Figura 11a)

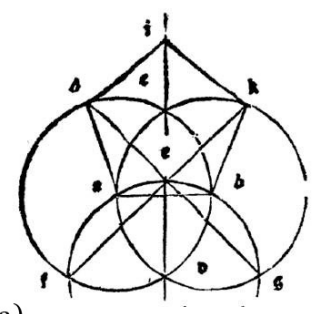

a)

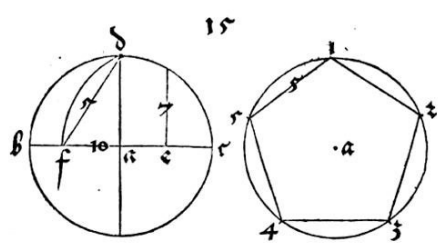

b)

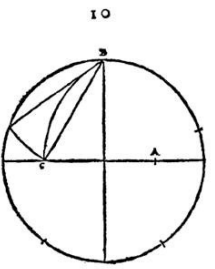

c)

Figura 11. Trazados pentágono en las geometrías prácticas. a) Roriczer M., b) A. Durero, c) J. Arfe. d). D. López Arenas.

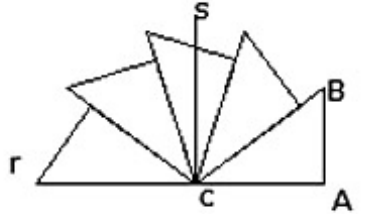

a)

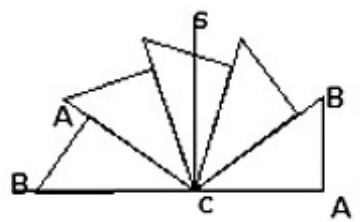

b)

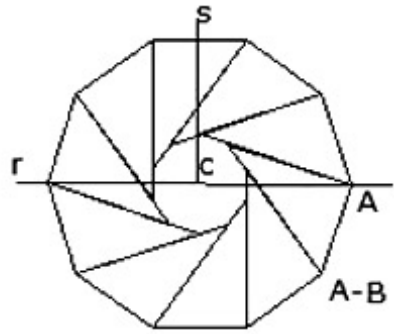

$\mathbf{r}$

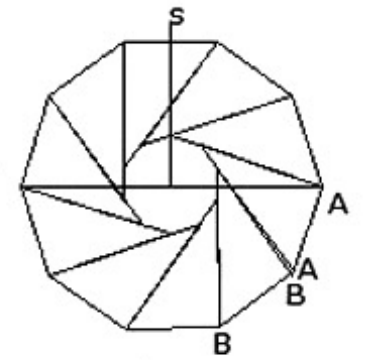

de Matthäus Roriczer (+c. 1495) (49) a partir de su lado. También en los primeros tratados renacentistas como el Underweysung der Messung (1525) (LII.15-16) (Figura 11b), de Albert Dürer (1471-1528) (50), el De varia commensvracion (1585) (L.I.Cap.II.10) (Figura 11c) de Juan de Arfe (1535-1603) (51) se realiza mediante la inscripción en la circunferencia, donde la construcción geométrica del pentágono es exacta, ya que dado un radio (100 u), se conocen todos los lados $(117,56 \mathrm{u})$, pudiéndose así construir mediante una escuadra de coordenadas decagonales $\left(36^{\circ}-54^{\circ}\right)$. No ocurre así con el trazado del Breve compendio de la carpinteria de lo blanco (1633), (Figura 11d) de López de Arenas (+c.1640) (Cap.I, fol. 1r-1 v) (52), ya que la proposición no tiene una solución precisa, con lo que cabría un pequeño ajuste del cartabón.

En la construcción de cualquier instrumento, existe una imprecisión, por lo que es necesario ajustar dicho utensilio. Así se traza una línea horizontal (r), en la que se sitúa el triángulo rectángulo $\mathrm{ABC}$, cuyo ángulo recto es el $\mathrm{A}$, disponiendo la hipotenusa $\mathrm{CB}$, sobre la recta y girando la escuadra sobre sí misma, se determinará que el vértice (A) debe estar sobre la línea horizontal. En caso contrario se deberá ajustar el ángulo reiterando el proceso. En algunas construcciones derivadas de las escuadras decagonales, la escuadra no necesita ajuste, puesto que es exacta (Figura 12a). Pero el sistema sugerido por un cartabón de cinco de López de Arenas sí necesitaría ajuste, puesto que la solución geométrica carecerá de precisión (7) (Figura 12b). También podríamos utilizar una escuadra del ábaco de la tumba de Saint-Ouen, o finalmente construir una escuadra de proporción (11/8), que es más precisa que el cartabón de cinco, o la del ábaco de Saint-Ouen (Figura 12c).

\section{LA ESCALERA DE SANTA CATALINA (1424) Y EL POLÍGONO DE TRECE LADOS}

La escalera de Santa Catalina se construye una vez acabadas las capillas radiales. El caragol sobiranes es conocido como caracol mayor de la catedral. La escalera se edifica a la vez que la última capilla radial, dedicada a Santa Catalina (1412-1424). En noviembre del año 1424 se erigen las puertas que cierran la escalera (53). La escalera de Santa Catalina, que es de caragol de botó redó, tiene $211,40 \mathrm{~cm}$ de diámetro, con un nabo de $21,60 \mathrm{~cm}$ y rosca de 14 tabicas de 22,00 cm, y trece rellanos trapezoidales (6,00, 96,00, $54,90,96,00 \mathrm{~cm})$. Tiene la especial característica que sus ocho primeros peldaños están construidos con dos piezas, teniendo éstas un ángulo recto, asimilables a una escuadra (Figura 13).

En la escalera de Santa Catalina la circunferencia se divide en 13 partes. Esta inscripción poligonal no aparece en los tratados matemáticos, ni en las geometrías prácticas de la época. Su construcción puede partir del ajuste de una escuadra, por interpolación entre la escuadra del hexágono (4/7) y la del heptágono (4/5) utilizando el ábaco de SaintOuen. Se construye así una escuadra (Ea) de proporciones (4/6) con lo que se procede a su rotación sobre su hipotenusa $(100 \mathrm{u})$, llegando a cierre con un desfase de tan solo $(4,5 \mathrm{u})$, sobre una longitud de $(628,31 \mathrm{u})$. Podemos también utilizar una escuadra $(E b)$ de proporciones (4,5/13), donde al cerrar la rosca, da un error de (o,62 u); por tanto, aunque menor que la $(E a)$, también requiere ajustarse (Figura 14). 

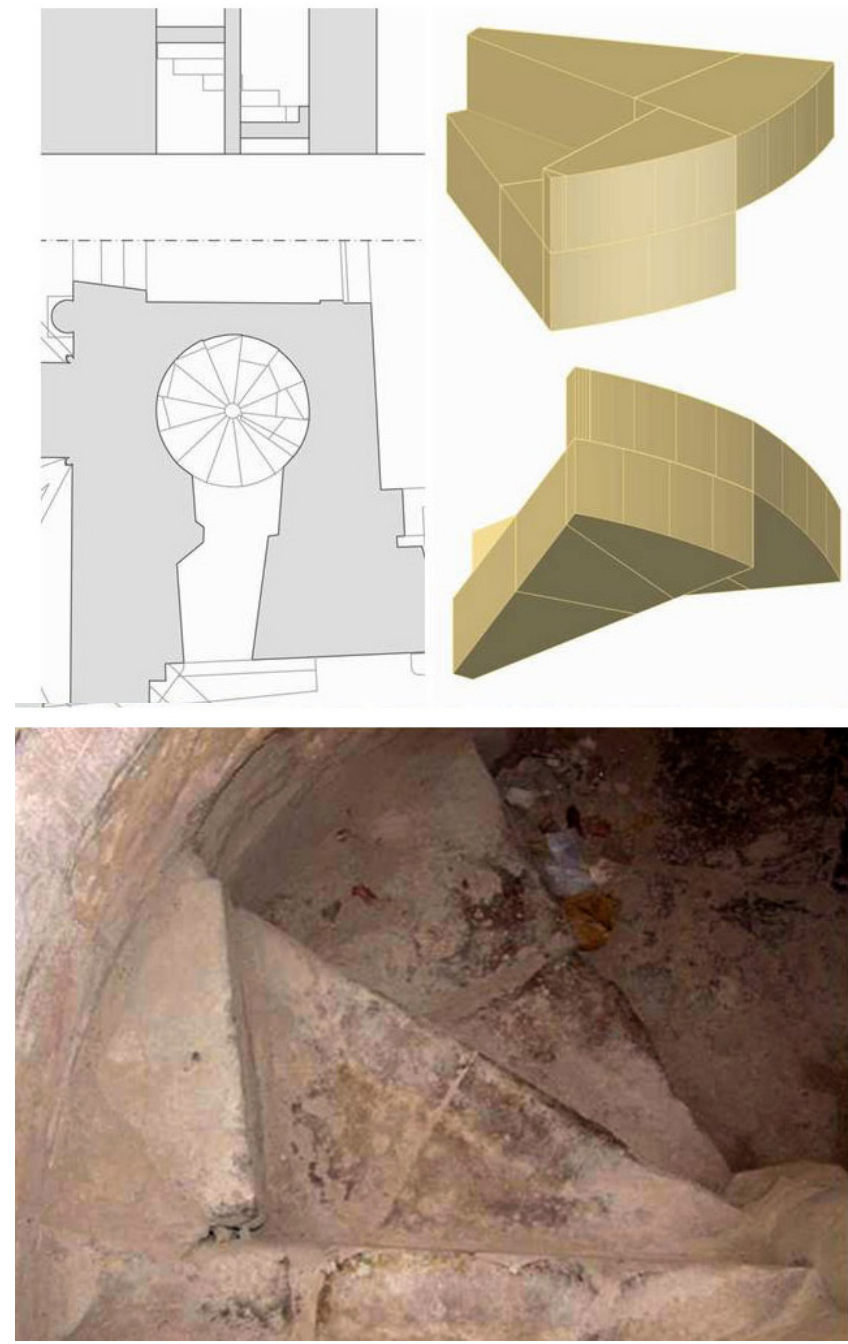

Figura 13. Escalera de caracol de Santa Catalina catedral Tortosa (1424), diámetro 211,40 cm.
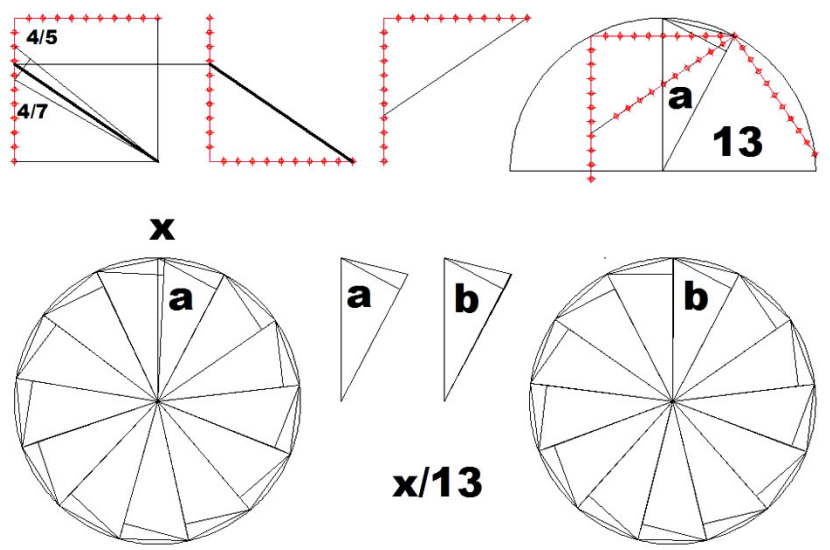

Figura 14. Construcción y ajuste de una escalera de caracol de 13 lados.

\section{CONCLUSIÓN}

En la escalera de caracol de Santa Catalina, las primeras piezas tienen un despiece en forma de escuadras, con lo que se divide el caracol en trece partes. En las fuentes directas no aparece una construcción geométrica para esta división poligonal. La utilización de la escuadra como ábaco de cálculo y plantilla, permite trazar este caracol. La proporcionalidad entre los catetos determina el ángulo interno de la escalera. La traslación reiterada de los catetos sobre la hipotenusa determina el giro del instrumento, a la vez que permite su ajuste. Estos métodos formarían parte de la geometria fabrorum, resultando muy eficaces para la división poligonal de 11, 13, 14, 17 o 19; las cuales no aparecen ni en las fuentes mathematicis, ni en los escasos códices de la Practica Geometriae.

\section{ABREVIACIONES}

BnF Bibliothèque Nationale de France.

CBM Carpentras Bibliothèque Municipale.

\section{REFERENCIAS}

(1) Lluis, J. (2009, 21-24 de octubre). Evolución constructiva de los pilares de una girola gótica. El concepto de homogeneidad del material versus resistencia. En Actas del Sexto Congreso Nacional Historia de la Construcción Valencia, (pp. 733-743). Madrid: Instituto Juan de Herrera.

(2) Fitchen, J. (1961). The construction of Gothic Cathedrals. A study of medieval vault erection, p.21. Oxford: Clarendon.

(3) Viollet-le-Duc, E. E. (1854-1868). Dictionnaire raisonné de l'architecture française du XIe au XVI ${ }^{e}$ siècle, vol. 5, pp. 295319. Paris: B. Bance.

(4) Choisy, A. (1883). L'art de bâtir chez les bizantins, pp. 46-47. París: Librairie de la Societé Anonyme de Publications Périodiques.

(5) Bechmann, R. (1991). Villard de Honnecourt. La pensée tecnique au XIIIe siècle et sa communication, pp. $146-148$. Paris: Picard Éditeur.

(6) Sarrade, M. T. (1986). Sur les connaissances mathématiques des bâtisseurs de cathédrales, p. 48 y pp. 52-54. Paris: Librairie du Compagnonnage.

(7) Nuere E. (2001). Nuevo tratado de la carpinteria de lo blanco, p. 68. Madrid: Ediciones Munilla-Leira.

(8) Baron, R. (1956). Hugonis de Sancto Victore Practica geometriae. Osiris, 12: 176-224, doi: http://dx.doi.org/10.1086/ 368598.

(9) Busard H.L.L. (1965). The Practica geometriae of Dominicus de Clavasio. Archive for History of Exact Sciences, 2(6): 520-575, doi: http://dx.doi.org/10.1007/BFo0324882.

(10) Bucher, F. (1980, noviembre). L'architecture vernacule ou l'empreinte du particularisme local. Histoire et Archéologie. Dossiers, p. 72.

(11) Vrijs, A. (1989). Catalogue. En Recht, R. (ed.) Les batîsseurs de cathédrales gothiques. Musées de la ville de Strasbourg, p. 372. Strasbourg. 
(12) Bucher, F. (1979). Architector. The Lodge books and sketchbooks of medieval architects. Vol. I., p. 226 y p. 235. New York: Abaris Book.

(13) Dürer, A. (1525). Underweysung der Messung, mit dem Zirckel und Richtscheyt: in Linien Ebnen vo gantzen Corporen, Libro I. Prop. 17. Nüremberg: Hieronymus Andreae.

(14) De L'Orme, P. (1576). L'architecture de Philibert de L'Orme, p. 123. Paris: Jérôme de Marnef et Guillaume Cavellat.

(15) Barbé-Coquelin de Lisle, G. (1977). El Tratado de Arquitectura de Alonso de Vandelvira. 2 vols, (fol.54). Albacete: Caja de Ahorros Provincial.

(16) Martínez de Aranda, G. (1986). Cerramientos y trazas de montea. Ed. Facsimilar, fol. 222 y fols. 221-224. Madrid: CEHOPU.

(17) Rabasa, E. (Ed). (2011). El manuscrito de cantería de Joseph Gelabert titulado Vertaderastraçes del Art de picapeder: transcripción, traducción, anotación e ilustración del texto y trazados, edición a cargo de Enrique Rabasa Díaz, pp. 106-112. Torrejón de Ardoz: C.O.A de les Illes Balears. Fundación Juanelo Turriano.

(18) Perouse de Montclos, J. M. (1985). La vis de Saint Gilles et l'escalier suspendu dans l'architecture française du xvi siécle. En L'escalier dans l'architecture de la Renaissance, pp. 83-91. Paris: Picard.

(19) Sanjurjo A. (2013). Experimentación geométrica y constructiva en piedra: algunos casos especiales de escaleras de caracol. Informes de la Construcción, 65(Extra-2): 35-48, doi: http://dx.doi.org/10.3989/ic.13.025.

(20) Benítez, P. (2013, 9-12 de octubre). Diseño y construcción del caracol de Mallorca de la iglesia del monasterio de Santa Cruz la Real (Segovia). En Actas del Octavo Congreso Nacional de Historia de la Construcción Madrid. Madrid: Instituto Juan de Herrera.

(21) Lisi, F. (1997). Timeo. En Platón Diálogos VI: Filebo, Timeo, Critias, pp. 125 y 261. Madrid: Gredos.

(22) García, V. (1998). Metafísica de Aristóteles $2^{\text {a }}$ Ed., pp. 472-473 y pp. 492-493. Madrid: Editorial Gredos.

(23) Heath, T.L. (1908). The Thirteen Books of Euclid's Elements. Vol. III, pp: 349-368. Cambridge: University Press.

(24) Giocondo, G. (Ed). (1511). M. Vitruvius per Iocundum solito castigatior factus, cum figuris et tabula, ut iam legi et intelligi potest, fol. 83v-85 r: Venecia: Tacuino.

(25) Faventino, M.C. (1540). De architectura compendiosissime tractans, p. 21. Paris: Michel de Vascosan.

(26) Lindsay, W.M. (1911). Isidori Hispalensis Episcopi. Etymologiarum sive originvm. Libri XX. Tomvs II. Libros XI-XX continens, p. 321. Oxford: Oxonii et Typographeo Clarendoniano.

(27) Migne J.P. (1851). Rabanus Maurus. De Universo Libri Viginti Duo. MPL111 (ooo9-o614B). Patrologiae cursus completus. Series latina, vol. 111, pp. 579-580. Paris: Jacques Paul Migne.

(28) Recht, R. (1980). Les termes techniques de l'architecture médiévale, Les bâtisseurs du Moyen-Âge. Histoire et Archéologie. Dossiers, pp. 84-90.

(29) Shelby, L. R. (1965). Medieval Masons' Tools II. Compass and Square. Technology and Culture, 6(2): 236-248, doi: http://dx.doi.org/10.2307/3101076.

(30) Wu N. (2000). Hugues Libergier and his Instruments. Nexus Network Journal, 2(1-2): 93-102.

(31) Sarrade, M. T. (1986). Sur les connaissances mathématiques des bâtisseurs de cathédrales, p. 50. Paris: Librairie du Compagnonnage.

(32) Sarrade, M. T. (1986). Sur les connaissances mathématiques des bâtisseurs de cathédrales, pp. 44-45. Paris: Librairie du Compagnonnage.

(33) Sene, A. (1970). Un instrument de précision au service des artistes du moyen âge: l'équerre. Cahiers de Civilitatión Médiévale, 13(52): 350-351, doi: http://dx.doi.org/10.3406/ccmed.1970.1874.

(34) Sene, A. (1972). Quelques instruments des architectes. Actes du congres de la Société des historiens médiévales de l'enseignment supérieur, pp. 42-43. Besanqon.

(35) Funck-Hellet, Ch. (1951). De la proportion de l'équerre des maîtres d'œuvre, p. 29. Paris: Éditions Vicent, Fréal \& Cie.

(36) Lassus, J.B. (1858). Album de Villard de Honnecourt. Architecte du XIII siècle, pp. 145-153. Paris: Imprimerie impériale.

(37) Erland-Branderburg, et.al. (1991). Villard de Honnecourt. Cuaderno Siglo XIII, p. 135. Madrid: AKal.

(38) Portet, P. (2004). Bertrand Boysset, la vie et les oeuvres techniques d'un arpenteur médiéval (v. 1355- v. 1416), pp. 255261. Paris: Éditions Le Manuscrit.

(39) Roriczer, M. (1999). Das Büchlein von der Fialen Gerechtigheit (fak. Regensburg 1486) und Die Geometria Deutsch (fak. Regensburg un 1487/88), pp. 56-60. Regensburg: Hürtgenwald Guido Pressler.

(40) Dürer, A. (1525). Underweysung der Messung, mit dem Zirckel und Richtscheyt: in Linien Ebnen vo gantzen Corporen, Libro II. Prop. 4. Nüremberg: Hieronymus Andreae.

(41) Almuni, V. (2007). La catedral de Tortosa als segles del gòtic. Vol. 2, pp. 472-474. Barcelona: Fundació Noguera. Col-lecció Estudis.

(42) Alanyà, J. (1998). Notícia de la Seu i Capítol a Tortosa a l'Arxiu de la Corona d’Aragó (segles XIV-XV). Anuario de Estudios Medievales, 28: 567-618, doi: http://dx.doi.org/10.3989/aem.1998.v28.io.6oo.

(43) Euclides. (1576). Los seis libros primeros de geometría de Evclides: traduzidos en lengua española por Rodrigo Çamorano Astrólogo y Mathemático y Cathedrático de Cosmographía por su Magestad en la casa de la Contratacion de Sevilla, pp. 71-79. Sevilla: Casa de Alfonso de la Barrera.

(44) Heath, T.L. (1908). The Thirteen Books of Euclid's Elements. Vol. III, pp: 88-111. Cambridge: University Press.

(45) Ptolomeo. (1578). Almagesto u obra claramente divina de la gran construcción de la matemática de feludiense alejandrino Claudio Ptolome, pp. 61-81. Venecia: Lucas Guarico.

(46) Toomer. G.J. (1984). Ptolemy’s Almagest, pp. 35-74. London: Duckworth.

(47) Woepcke, M. F. (1855). Recherches sur l'historie des Sciences mathématiques chez les orientaux, dápres des traités inedits Arabes et Persans. Deuxième siecle. Analyse et extrait d'un recueil de constructions géométriques par Abûl Wafa. Journal asiatique, $5:$ 309-359 (CII.3) y (CIII. 9-11). 
(48) Aghayani-Chavoshi. (2010). Ketâb al-nejârat (Sur ce qui est indispensable aux artisans dans les constructions géométriques), W43. Tehran: Written Heritage Research Centre \& Institut Français de Recherche en Iran.

(49) Roriczer, M. (1999). Das Büchlein von der Fialen Gerechtigheit (fak. Regensburg 1486) und Die Geometria Deutsch (fak. Regensburg un 1487/88). Regensburg: Hürtgenwald Guido Pressler.

(50) Dürer, A. (1525). Underweysung der Messung, mit dem Zirckel und Richtscheyt: in Linien Ebnen vo gantzen Corporen. Nüremberg: Hieronymus Andreae.

(51) Arfe, J. (1585). Ioan de Arphe y Villafañe (1535-1603). De varia commensuracion para la escultura y architectura. Sevilla: Andrea Pescioni y Juan de León.

(52) Lopez de Arenas, D. (1633). Breve Compendio de la Carpintería de lo blanco y tratado de alarifes, con la conclusión de la regla de Nicolás Tartaglia, y otras cosas tocantes son la Iometria, y puntas de compás. Sevilla: Luis Estupiñan.

(53) Almuni, V. (2007). La catedral de Tortosa als segles del gòtic. Vol. 2, pp. 152-157. Barcelona: Fundació Noguera. Col·lecció Estudis. 